\title{
Commentary
}

\section{Reply to the response of Dr. Bachour to our manuscript "Ultrasound elastography for the detection of capsular fibrosis in breast implants: First results"}

\author{
V. Hoesl* \\ Center of Plastic, Aesthetic Hand and Reconstructive Surgery, University Hospital Regensburg, \\ Regensburg, Germany
}

Dear Editor,

We thank Dr. Bachour for the response [1] to our manuscript "Ultrasound elastography for the detection of capsular fibrosis in breast implants: First results" [2].

We agree completely that the Baker score is a subjective classification of capsular contracture. In order to create stable conditions, the examination and evaluation of all included patients were performed by an experienced physician.

The relationship between the subjective clinical examination and the objective examination was evaluated using ultrasound. We assumed that the same experienced investigator would evaluate the clinical findings in a very similar way. In order to be able to compare the results more easily, independent of the investigator, objectifiable methods are essential. Therefore, we compared both methods.

With regard to the surgical procedure, a capsulectomy and implant removal was performed before the insertion of polyurethane (PU)-coated breast implants.

This study investigates capsule formation after the insertion of polyurethane (PU) coated breast implants that are not normally used in our approach as primary implants. It is certainly interesting to perform the study in patients after the insertion of the primary implant, however in this study, we intended to find out whether a capsule can form again after PU-coated implants.

The study presents our first results on the evaluation of capsular fibrosis after the insertion of PUcoated breast implants after 36 months. Capsule formation occurs most often in the first 3 years and in our preliminary study we could see a relevant and significant difference. To confirm these initial results, a further examination of the patients is planned after a longer period of time. The results of this study will motivate the examination of a larger group of patients observed over a longer period of time. In this context, an objective classification of capsular contracture may possibly be developed

\footnotetext{
*Corresponding author: V. Hoesl, MD, Center of Plastic, Aesthetic Hand and Reconstructive Surgery, University Hospital Regensburg, Franz-Josef-Strauß-Allee 11, 93053 Regensburg, Germany. Tel.: +49 941944 6763; Fax: +49 941944 6948; E-mail: Vanessa.hoesl@ukr.de.
} 


\section{References}

[1] Bachour Y. Response to: Ultrasound elastography for the detection of capsular fibrosis in breast implants: First results. Clin Hemorheol Microcirc. 2021;77(3):351-352. DOI:10.3233/CH-201012.

[2] Jung E, Hösl V, von Fraunberg S, Jung F, Prantl L. Ultrasound elastography for the detection of capsular fibrosis in breast implants: First results. Clin Hemorheol Microcirc. 2021;77(3):247-257. DOI:10.3233/CH-200875. 